\title{
An Angular Distribution of Three Photon Ionization in Presence of Multiple Laser Beams
}

\author{
Nilam Shrestha ${ }^{1}$ and Jeevan Jyoti Nakarmi ${ }^{2}$ \\ ${ }^{1}$ Department of Physics, Tri-Chandra multiple campus, Tribhuvan University,Kathmandu \\ ${ }^{2}$ Central Department of Physics, Tribhuvan University, Kirtipur \\ Corresponding author: Nilam Shrestha (nilamspradhan@gmail.com)
}

\begin{abstract}
We have studied the effect of phase difference between different multiple beams on the photoelectron angular distribution in three photon ionization of atomic hydrogen by using Dalgarno-Lewis methods. It has been found that both total and photo-electron angular distribution vary with laser intensity, phase and polarization of different beams. Intensity dependance of corresponding assymetry parameters has also been studied. We have shown that this variation with laser intensity is caused by two effects: the effect of phase and polarization of different beams. Asymmerties of azimuthal angular distribution are emphasized.
\end{abstract}

Keywords: Angular distribution, transition probability and cross-section

\section{Introduction}

In his famous discovery of the photoelectric effect, Albert Einstein reasoned that photons might ionize an atom only if they had energy greater than a particular threshold energy corresponding to the ionization energy of the atom. In 1929, Maria Göppert-Mayer predicted theoretically that an atom might absorb two or more photons simultaneously, thus allowing an electron to transition to states unreachable by single photon absorption. An atom absorbing multiple photons simultaneously might be ionized by photons with energies less than the threshold energy of which Einstein spoke. Because any observable effect of this phenomenon could not be possible without a very intense beam of radiation, his prediction could not be investigated in detail until the construction of the first laser in 1960. At the sufficiently high photon flux densities, however, there is an increasing probability for simultaneous absorption of two or even more photons by the atom and hence the process is called Multiphoton Ionization. In three photon process, where three photons of same frequency are simultaneously absorbed in one event and make a transition from initial state $|g\rangle$ to and excited state $|f\rangle$ allowed by three photon selection rules [e.g., Townsend, 2000].

We consider light incident along the $\mathrm{z}$ axis interacting with an unpolarized hydrogen atom at the origin via the electric dipole interaction and ejecting a photo electron along a direction described by the angle $\theta$ (Figure 1). In case of $\mathrm{N}$ - photon ionization of the atoms, the photoelectron angular distribution can be described by the general formula of differential ionization cross-section given by

$$
\frac{d \sigma^{(N)}}{d \Omega}=\sum_{i=0 \text { to } N} a_{i} \cos ^{2 i} \theta
$$




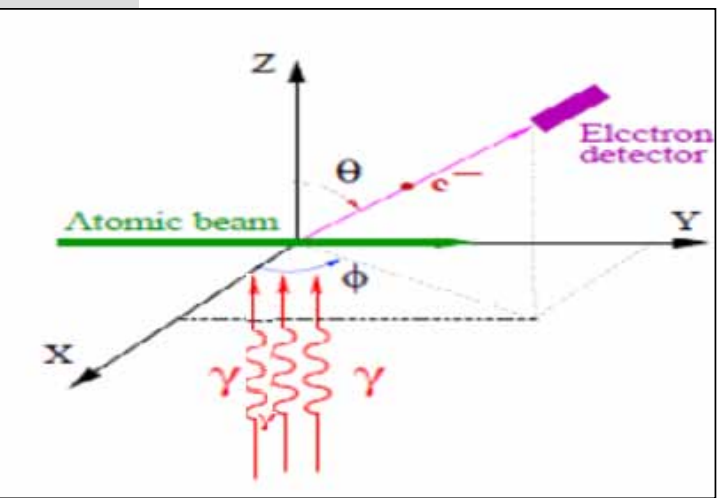

Figure 1. Geometry accepted in theoretical consideration of the angular distribution[e.g., Koval, P., 2004]

Where angle $\theta$ is the angle between the direction of ejected electrons and polarization of laser field, and $a_{i}$ is the coefficient depending on the properties of the initial and intermediate states of atom, partial waves of emitted electron and the radiation field. We have presented this coefficient in matrix formalism with taking into account both atomic structure and dynamics of process.

\section{Angular Distribution}

Photoelectron angular distributions generally contain more information about the dynamics of a process than its total probability. Polarization describes the orientation of electric field vector when the electromagnetic wave is propagating in space. The theoretical explanation on the polarization dependence of multiphoton ionization by Lambropoulos [e.g.,Lambropoulos,, 1972] shown that total cross-section of two-and three-photon ionization of one-electron atom depend on the polarization of incident light. Expressions are derived for the ratio of the cross-section for circularly polarized to that for linearly polarized light and their maximum values are obtained. Polarization dependence are best understood by the relation connecting the cross-section of linear and circular polarization[e.g., Delone et al, 1999]. Experimental was done for four photon in Cesium atom by inserting a halfwave plate between Glan prism and focussing lens [e.g., Petite et al.,1984].

The electronic orbital presented here represent a volume of space within which an electron would have a certain probability of being based on particular energy states and atoms. For example, as simplicity lowest energy state hydrogen atom, the electrons are most likely to be found within a sphere around a nucleus of an atom. In a higher energy state, the shape becomes lobes and rings, due to the interaction of the quantum effects between different atomic states. These shapes continue on infinitely getting ever more lobes or rings on them. The shape of the orbital depends upon many factors. The most important are the quantum number with the particular energy states $\quad(n, l, m)$. Although $l=0$ and $m=0$ orbital looks like simple sphere, regardless of the $\mathrm{n}$ values, this is not actually the case.

For $n \geq 4, l=0$ and $m=0$, orbital showing that, it is really a concentric sphere. The atomic orbital model thus predicts line spectra, which are observed experimentally. The angular distribution represented here as a polar diagram is calculated in $x-y$ plane in which all multiple laser beams are vibrating. The photon hvis incident along the $z$ axis. The azimuthal angle of the photoelectron with respect to $\mathrm{x}$-axis is indicated by $\mathrm{f}$. All Legendre polynomials have at and, so that in general the angular distributions have two maxima at those angles and an oscillatory dependence between them. 
is Legendre polynomial of order $l$ [e.g., Dixit, 1983]. Electron angular distribution produced in resonance enhanced two-photon ionization of $\mathrm{H}+2$ molecular ion using-ultra-short laser pulse have been theoretically reported [e.g., Selsto et al. , 2007]. The probability distribution of the electron, can be interpreted as the electronic charge distribution. Many atomic properties,however, depend mainly on the form of the angular distribution.

\section{Differential Cross-section}

The differential rate for three photon absorption in a. u. has expression [e.g., Fifirig et al., 2002]

$$
\frac{d \sigma^{(3)}}{d \Omega}=\frac{\alpha}{2 \pi}\left(\frac{1}{2 I_{0}}\right)\left|M_{f g}(3)\right|^{2} a_{0}^{2} \omega k_{f}
$$

Where, $I_{0}=7.019 \times 10^{16} \mathrm{~W} / \mathrm{m}^{2}, k_{f}$ momentum of ejected electron, $\alpha$ the fine structure constant, $\omega$ laser frequency and $\left[M_{\text {(3) }}\right]^{\text {is }}$ transition amplitude from initial ground state to final excited state for three photon.

$M_{f g}^{(3)}=\sum_{m n} \frac{\left\langle f\left|\left(\hat{\varepsilon}_{l \cdot} \cdot \vec{r}\right)\right| m\right\rangle\left\langle m\left|\left(\hat{\varepsilon}_{k} \cdot \vec{r}\right)\right| n\right\rangle\left\langle n\left|\left(\hat{\varepsilon}_{j} \cdot \vec{r}\right)\right| g\right\rangle}{\left(\omega_{m g}-2 \omega\right)\left(\omega_{n g}-\omega\right)}$

Here, $l, \mathrm{k}$ and $\mathrm{j}$ represent the number of beams used. The summation over the complete spectrum contained in above formula is difficult to be performed. Several approaches and approximation techniques have been proposed to perform such a summation. Among them Dalgarno-Lewis(DL) method has been widely used [e.g., Dalgarno and Lewis, 1986]. The idea of DL method is to define an axuliary operator such that evaluation of intermediate sum is not needed.
Therefore $M_{f g}^{(3)}$ can be made simpler by using Dalgarno-Lewis method and solved by assuming one more operator in addition to twophoton process[e.g., Shrestha, 2013].

\section{Three-photon selection rule}

Transition amplitude are obtained by using, recursion relation of the Lengendre polynomial with $x=\left(k_{f} \cdot \vec{r}\right)$ and after performing angular integration. The angular separation of the function can be performed in terms of the spherical harmonics using the orthogonality property of the Legendre Polynomials.

$M_{f g}^{(3)}=\int(2 l+1) P_{l}(k f \cdot \vec{r})(i) R_{k f}(r)\left(\hat{\varepsilon}_{l} \cdot \vec{r}\right) \times$
$\left[\left(\frac{3}{2}\left(\hat{\varepsilon}_{k} \cdot \vec{r}\right)\left(\hat{\varepsilon}_{j} \cdot \vec{r}\right)-\frac{1}{2}\left(\hat{\varepsilon}_{k} \cdot \hat{\varepsilon}_{j}\right) r^{2}\right) g_{2}+\left(\hat{\varepsilon}_{k} \cdot \hat{\varepsilon}_{j}\right) g_{0}\right] \psi_{g} d \tau$

Where $\mathrm{g}_{0}$ and $\mathrm{g}_{2}$ are the solution to Dalgarno operators.

We have from spherical addition theorem [e.g., Devananthan, 2002]

$$
P_{l}\left(k_{f} \cdot \vec{r}\right)=\frac{4 \pi}{2 l+1} \sum_{l m} Y_{l m}\left(k_{f}\right) Y^{*}{ }_{l m}(\vec{r})
$$

\section{Separating angular and radial part}

$$
\begin{aligned}
& \left.\left.M_{f g}^{(3)}=3(i)\left[\frac{4 \pi}{15}\left(\hat{k}_{f} \cdot\left(\hat{\varepsilon}_{i}\right)_{k} \cdot \hat{\varepsilon}_{k} \cdot \hat{\varepsilon}_{j}\right)+\hat{k}_{f} \cdot\left(\hat{\varepsilon}_{k} \cdot \hat{\varepsilon}_{j} \cdot \hat{\varepsilon}_{l}\right)+\hat{k}_{f} \cdot\left(\hat{\varepsilon}_{k} \cdot \hat{\varepsilon}_{j} \cdot \hat{\varepsilon}_{l}\right)\right)-\frac{4 \pi}{9} \frac{4 \pi}{15} \hat{\varepsilon}_{k} \cdot \hat{\varepsilon}_{j} \cdot \hat{\varepsilon}_{l}\right)\right] \\
& \left.\times] R_{k f l}(r) r^{5} g_{2} e^{-r} d r+4(i) \pi k_{f} \cdot\left(\hat{\varepsilon}_{k} \cdot \hat{\varepsilon}_{j} \cdot \hat{\varepsilon}_{l}\right)\right] R_{k f f}(r) g_{0} r^{3} e^{-r} d r
\end{aligned}
$$

For $l=1$

$$
\begin{gathered}
\int P_{l}\left(k_{f} \cdot \vec{r}\right)\left(\hat{\varepsilon}_{j} \cdot \vec{r}\right) d \Omega=4 \pi / 3\left(\hat{k}_{f} \cdot \hat{\varepsilon}_{j}\right), \text { if } l=1 \\
=0, \text { if } l \neq 1
\end{gathered}
$$

For $l=3$

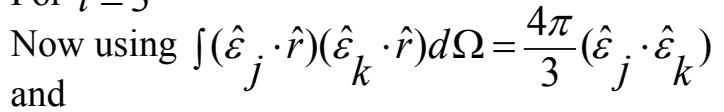


$\int\left(\hat{\varepsilon}_{j} \cdot \hat{\varepsilon}_{k}\right) d \Omega=4 \pi\left(\hat{\varepsilon}_{j} \cdot \hat{\varepsilon}_{k}\right)$

Separating angular and radial part

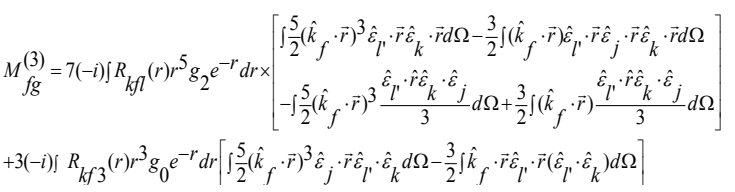

$M_{f g}^{(3)}=7(-i) \int R_{k f l}(r) r^{5} e^{-r} d r \mid P_{3}\left(\hat{k}_{f} \cdot \vec{r}\right)\left(\hat{\varepsilon}_{j} \cdot \vec{r}\right)\left(\hat{\varepsilon}_{l} \cdot \vec{r}\right)\left(\hat{\varepsilon}_{k} \cdot \vec{r}\right)-\frac{\hat{\varepsilon}_{l} \cdot \hat{\varepsilon}^{\prime} k}{3} g_{2} d \Omega$

$\left.+3(-i) \int R_{k f 3}(r) r^{3} e^{-r} d r+\int P_{3}\left(\hat{k}_{f} \cdot \vec{r}\right) \hat{\varepsilon}_{j} \cdot \vec{r}\left(\hat{\varepsilon}_{l l} \cdot \vec{r}\right) \hat{\varepsilon}_{k}\right) g_{0} d \Omega$

The radial parts of integrals are solved [e.g., Radhakrishanan and Thyaullathil, 2004]. We have discussed only the angular parts (angular distribution in terms of polar plots) with phase dependence.

\section{Results and Disscussion}

Wehaveplotted differentialionizationcrosssection of three- photon ionization produced by an electromagnetic field containing, single, two and three beams with varying phase difference propagating along the same direction taken as $\mathrm{z}$ axis of coordinates system. We have used polar angle $\theta=\pi / 2$ and wavelength of the photon $=$ $12 \times 10^{-8} \mathrm{~m}$. The angular distribution represented here, as polar diagrams, is calculated in the $\mathrm{x}$ - y plane, in which all three laser beams are vibrating. Angular distribution $\left|\mathrm{Y}_{\mathrm{lm}}(\theta, \varnothing)\right|^{2}$ is axially symmetric with respect to azimuthal angle $f$, which we have varied from 0 to $2 \pi$. The graphical output is placed immediately after its corresponding input command as it appears in mathematica notebooks. Arbitrary units are used in these graphs. In the present work we have found that photo ionization with different beams of same frequency gives quite different pattern of angular distribution. The figure(2) is for two-photon two beams via perturbative calculation, and our result figure (3)is obtained from non-perturbative by Dalgarno-Lewis method, both show the asymmetries in multi photonionization. Thus we found the same result as displayed below, figure (4)for different phase angles of arbitrary beams.

The thick solid line, the thin solid line and dashed line show the changes in alignment by
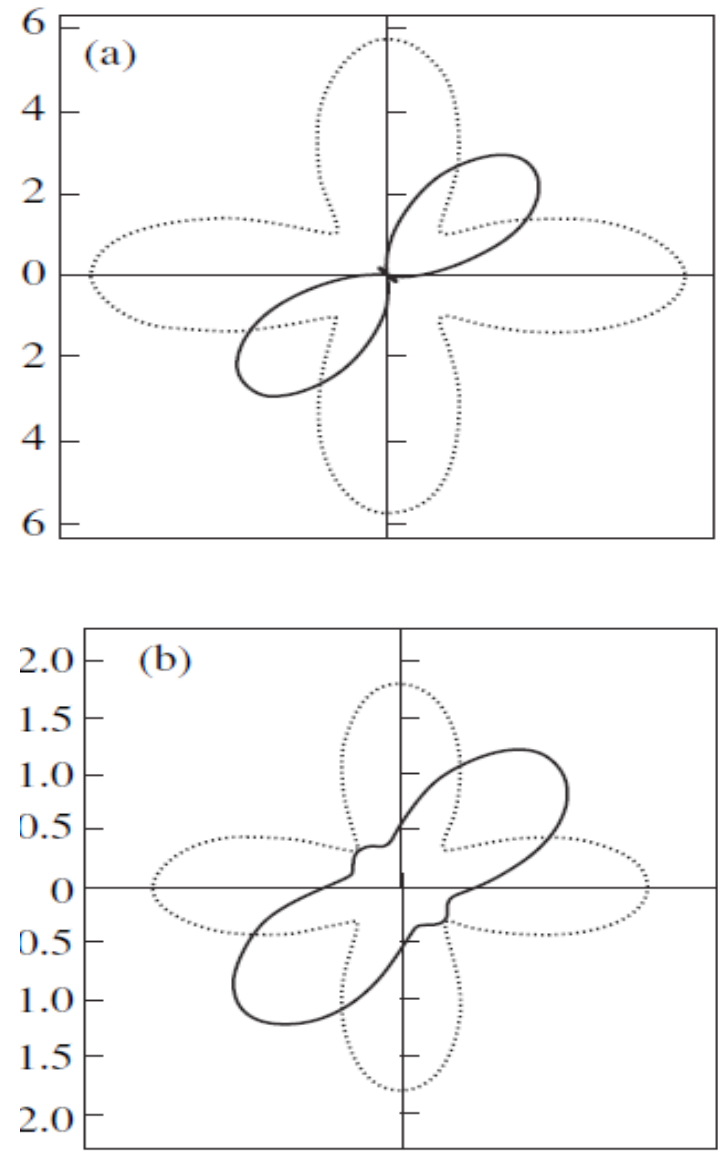

Figure 2. Asymmetries in two-photon ionization [e.g., Fifirig and Store, 2002] 


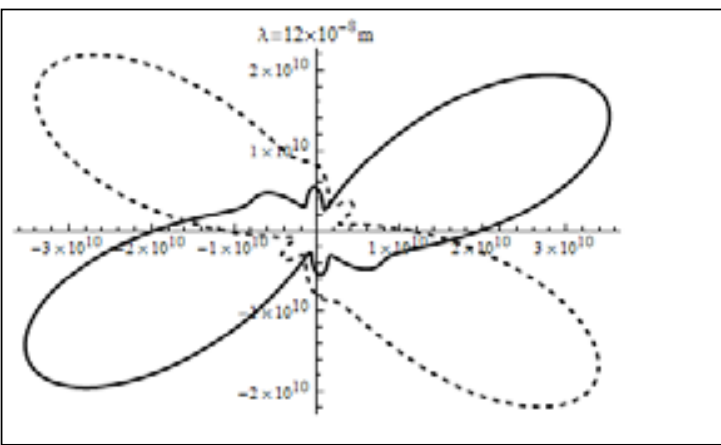

Fig a. Three photon two beams

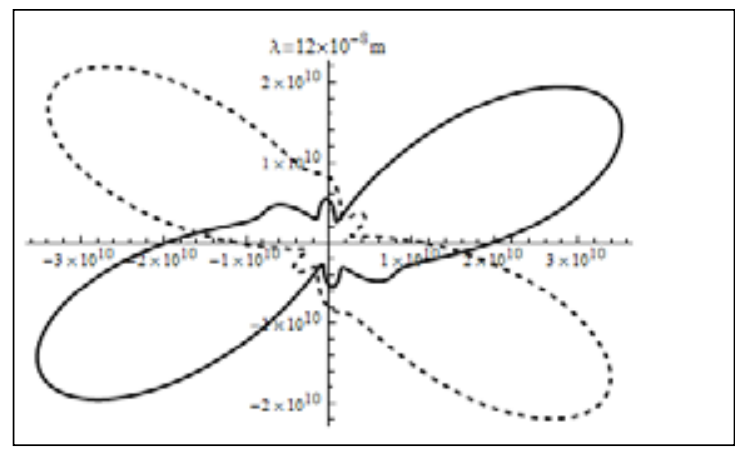

Fig b. Three photon three beams

Figure 3. Asymmetries in three -photon ionization changing phases of different beams. Thus the alignment of azimuthal distribution is strongly modified by phase difference $\delta$ between two beams. For the particular value of the phase difference $(\delta=\pi / 2)$ the magnitude and the

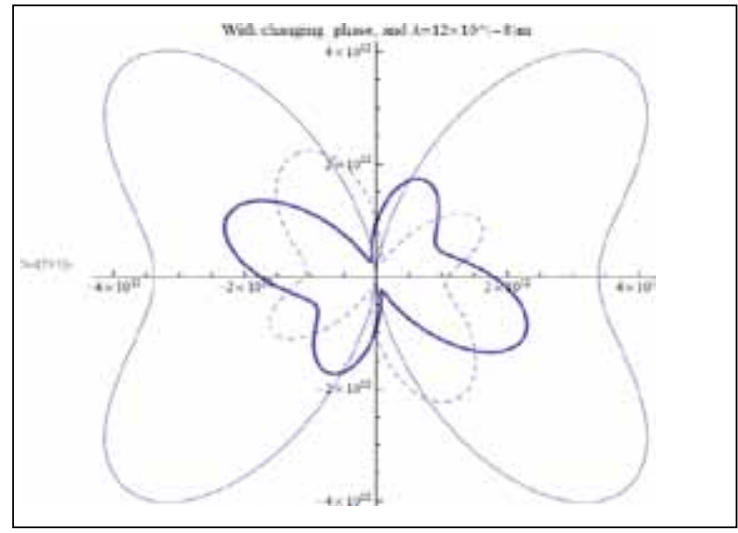

Fig c. Three photon four beams

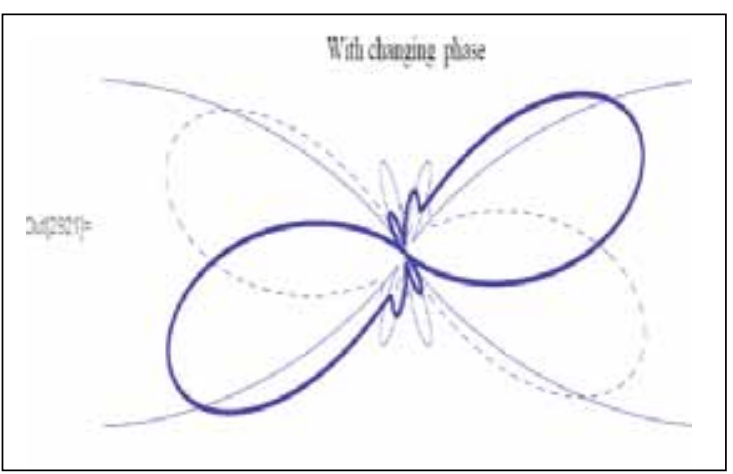

Fig d. Three photon five beams

Figure 4. Asymmetries in three -photon ionization for more beams

alignment of the azimuthal angular distribution for left circular polarization are quite different than that for right circular polarization.

\section{Conclusions}

We studied the differential ionization cross-section and angular distribution of MPI of hydrogen atom based on Dalgarno-Lewis and third order perturbation theory. The polar plots of Figure (3) and Figure (4) show that differential ionization cross-section of atomic hydrogen vary by changing the phase difference of multiple laser beams of same frequencies but different polarization. Calculations obtained within the electric dipole approximation and by including all multiple beams are separately displayed. Therefore there are asymmetries of angular distribution of multiphoton ionization. This result shows that the angular distribution of MPI with multiple beams is more isotropic than the one with single photon with ultra-short pulse. Finally, in order to give a more practical account of the angular distribution function, we expanded it with Legendre polynomials and we displayed the results. Further studies are in 
progress to investigate the polarization effect of scattered photon on MPI in presence of multiple laser beams.

\section{Acknowledgments}

The authors would like to express their gratitude to supervisor Dr. Lok Narayan Jha, CDP, Kathmandu. A sincere appreaciation goes to Prof. Ramesh Babu Thayyullathil and Dr. Radhakrishanan, CUSAT, Kerala, India. Uuniversity grant commssion and India-Nast bilateral exchange program are also acknowlege for their financial support. Editor N. P. Chapagain thanks the reviewers for their assistance inevaluating this paper.

\section{References}

Dalgarno, A. and J. T. Lewis (1956), Proc. Phys. Soc. A: 69, 628-.631.

Delone, N. B. and V. Krainov (1999), Multiphoton Processes in Atoms, 190-199.
Devananthan, V. (2002), Angular momentum techniques in quantum mechanics, Kluwer Academic Publishers, 115-126.

Dixit, S. N. (1983), J. Phys., 16(B), 1205-1210.

Fifirig M.and M. Stroe, (2002), Laser physics, 12, 963-969.

Koval, P. (2004), Thesis, Two photon ionization of atomic inner shells, University of Kassel, 25-26.

Lambropoulos, P. (1972), Phy. Rev. lett, 28, 585589.

Petite, G. , F. Fabre, P. Agostini (1984), Phy. Rev., 29(A5), 2677-2689.

Radhakrishnan, R. and T. Ramesh babu (2004)., Phy. Rev. A 69, 033407-033412.

Selstø, S., Alicia P., Fernández J., and Fernando M. (2007), Phy. Rev., 75(A), 033419-033424.

Shrestha N. (2013), Nepal Journal of Science and Technology (NAST), 14(2), 165-170.

Townsend, S. J. (2000), A modern Approach to quantum mechanics, University Science books print, 93-105. 